\title{
Deficiency of Interleukin-1 Receptor Antagonist Promotes Spontaneous Femoral Artery Aneurysm Formation in Mice
}

Kikuo Isoda, ${ }^{*}$ Manabu Kitagaki, ${ }^{\dagger}$ Tomiharu Niida, ${ }^{*}$ Harumi Kondo, ${ }^{*}$ Osamu Matsubara, ${ }^{\ddagger}$

Makoto Kikuchi, ${ }^{\dagger}$ Fumitaka Ohsuzu, ${ }^{*}$ and Takeshi Adachi*

From the Departments of Internal Medicine 1,* Medical Engineering, ${ }^{\dagger}$ and Basic Pathology, ${ }^{\ddagger}$ National Defense Medical College, Tokorozawa, Japan

Femoral artery aneurysms (FAAs) are very rare, and their natural history is not well understood. In this study, we sought to analyze the pathogenesis of inflammatory FAAs in interleukin-1 receptor antagonist-deficient $\left(\mathrm{IL}-1 \mathrm{Ra}^{-/-}\right) \mathrm{B} 6$ mice. Systolic arterial pressures and plasma lipid levels of $I L-1 \mathrm{Ra}^{-/-}$mice and wild-type (WT) mice did not differ significantly. However, $I L-1 \mathrm{Ra}^{-/-}$mice spontaneously developed fusiform FAAs. Real-time PCR of 9-month-old $I L$ $1 \mathrm{Ra}^{-/-}$mice revealed significantly increased mRNA levels of IL-1 $\beta$ (6.6-fold), tumor necrosis factor- $\alpha$ (TNF- $\alpha$ ) (12.4-fold), and matrix metalloproteinase-9 (6.0-fold) compared with WT mice. Histological analysis revealed numerous inflammatory cells around the FAAs in $I L-1 R^{-/-}$mice, and elastin staining showed destruction of both the internal and external elastic lamina in $I L-1 R a^{-/-}$mice. Afterward, macrophage function was studied. After lipopolysaccharide $(1 \mu \mathrm{g} / \mathrm{mL})$ stimulation, IL-1Ra-deficient macrophages produced much higher levels of TNF- $\alpha$ than those from WT mice. Finally, we performed bone marrow cell transplantation. FAAs with many inflammatory cells in the adventitia were detected in several WT mice that received bone marrow cells from $I L-1 \mathrm{Ra}^{-1-}$ mice (44\%), but not from WT mice ( $0 \%)$. Our study is the first to demonstrate that IL-1Ra deficiency in inflammatory cells disrupts immune system homeostasis and induces inflammatory FAAs in $I L-1 \mathrm{Ra}^{-/-}$ B6 mice. We believe that these mice will provide much information about the natural history and management of FAAs. (Am J Pathol 2012, 180: 1254-1263; DOI: 10.1016/j.ajpath.2011.11.028)
Arterial aneurysms represent a complex degenerative disorder involving chronic arterial wall inflammation and destructive remodeling of structural connective tissue. ${ }^{1}$ The relative balance of pro- and anti-inflammatory cytokines likely controls inflammation in the aneurysm wall. ${ }^{2,3}$ Previous studies demonstrated abundant expression of many cytokines, including IL- $1 \beta$, in the aneurysm wall. ${ }^{4,5}$ The IL-1 receptor antagonist (IL-1Ra) negatively regulates $\mathrm{IL}-1$ signaling by binding and blocking the functional receptor without activating it. ${ }^{6}$ Thus, IL-1Ra plays an anti-inflammatory role in acute and chronic inflammation, ${ }^{7}$ but showed no direct evidence implicating endogenous IL-1Ra in aneurysm formation.

Femoral artery aneurysms typically occur at an older age, ${ }^{8,9}$ and those are often bilateral and may associate with multiple aneurysms. A previous report showed that $36 \%$ of patients with a femoral artery aneurysm also had a contralateral femoral artery aneurysm, and $69 \%$ had multiple aneurysms. ${ }^{10}$ Femoral artery aneurysms are very rare, and most studies investigating these aneurysms are published as case reports. ${ }^{8,9,11-13}$ Consequently, the natural history of femoral artery aneurysms is poorly understood.

We demonstrate here for the first time that IL-1Ra-deficient $\left(I L-1 R a^{-/-}\right)$mice $^{14-16}$ develop spontaneous femoral artery aneurysms, and the mice represent the first animal model for femoral artery aneurysm. We believe these mice will provide much information about the natural history and management of femoral artery aneurysms.

\section{Materials and Methods}

\section{Animals}

The experimental protocol used in the study conforms with the Guide for the Care and Use of Laboratory Ani-

Supported by National Defense Medical College grants H14 (K.I.) and H18 (K.I.).

Accepted for publication November 8, 2011.

K.I. and M.K. contributed equally to this work.

Address reprint requests to Kikuo Isoda, M.D., Ph.D., Internal Medicine 1, National Defense Medical College, 3-2 Namiki, Tokorozawa, Saitama 359-8513, Japan. E-mail: isoda@ndmc.ac.jp. 
mals published by the US National Institutes of Health (NIH Publication No. 85-23, revised 1996) and was approved by the National Defense Medical College Board for Studies in Experimental Animals.

$I L-1 R a^{-1-}$ mice were generated by replacing all exons of the secreted form with the neo gene by homologous recombination as described. ${ }^{14-16}$ Throughout the experiment, the mice consumed a normal diet.

\section{Blood Pressure Measurement}

Systolic blood pressure was measured by the tail-cuff method (MK-2000; Muromachi Kikai, Tokyo, Japan) without anesthesia.

\section{Plasma Lipid Measurements}

After fasting the mice for 7 hours, plasma total cholesterol, and high-density lipoprotein cholesterol levels were measured by enzymatic assays as previously described by Hedrick et al. ${ }^{17}$

\section{Leukocyte and Platelet Counts}

Leukocyte and platelet counts were measured in blood using hematology analyzer Vet ABC Animal Blood Counter and ABC VET LMGE Reagent Pack (Horiba ABX, Kyoto, Japan).

\section{Tissue Preparation and Histology}

After measuring tail-cuff systolic blood pressure, male mice were euthanized with pentobarbital (dose: $100 \mathrm{mg} /$ $\mathrm{kg}$ ), and perfused with $0.9 \% \mathrm{NaCl}$, followed by $4 \%$ paraformaldehyde. Following perfusion, the femoral artery was harvested, fixed overnight in 4\% paraformaldehyde, embedded in optimal cutting temperature compounds and sectioned (10 $\mu \mathrm{m}$ thickness). Samples were routinely stained with H\&E, Picrosirius red, Masson trichrome, or elastica van Gieson. Smooth muscle cells (SMCs) were visualized with $\alpha$-smooth muscle actin staining (Roche, Indianapolis, IN), and apoptotic cells were visualized with cleaved caspase-3 antibody (Cell Signaling Technology, Danvers, MA). Granulocytes were visualized with monoclonal Ly-6G (BD Biosciences/Pharmingen, Franklin Lakes, NJ), mouse macrophages were visualized with monoclonal Mac-3 (BD Biosciences Pharmingen), and T cells were visualized with monoclonal CD4 (BD Biosciences Pharmingen). We also checked the expression of tumor necrosis factor- $\alpha$ (TNF- $\alpha$ ) (Abcam, Cambridge, UK) and matrix metalloproteinase-9 (MMP-9) (Abcam). Endogenous peroxidase was blocked by incubation with $3 \% \mathrm{H}_{2} \mathrm{O}_{2}$ in methanol for 5 minutes. Slides were incubated with normal swine serum (Vector Laboratories, Burlingame, CA) for 10 minutes and then with primary antibody overnight at $4^{\circ} \mathrm{C}$. The secondary antibody was the biotinylated antibody (Dako, Glostrup, Denmark). We visualized the sections with a Vectastain $A B C$ kit (Vector Laboratories), using diaminobenzidine as the substrate. The nuclei were counterstained with Mayer's hematoxylin solution.

\section{Morphometry}

We removed femoral artery specimens from mice at 7,8 , and 9 months of age and used 20 equally spaced cross sections in all mice for quantification. The images were digitized to perform morphometric analysis using ECLIPS LV100 microscopy (Nikon, Tokyo, Japan), and we measured the maximal vessel area (the area within the media/ adventitial border) and lumen area using NIS-Elements (Nikon). The intimal + medial area was calculated by subtracting the lumen area from the vessel area. Cleaved caspase-3-positive nuclei were quantified, and apoptotic indexes (apoptotic nuclei/total nuclei $\times 100$ ) were calculated. $\alpha$-SMA-positive areas were calculated in the cross sections of the media of femoral arteries and expressed as a percentage of the medial area. MMP-9- and TNF$\alpha$-positive areas were calculated in the longitudinal sections of femoral arteries and expressed as a percentage of the total area. For quantification of the positive area, captured photomicrographs were transferred into an image analysis system (ImagePro Plus 5.1; Media Cybernetics, Bethesda, MD). A color-threshold mask for immunostaining was established to detect reddish brown (positive) staining, and the same threshold was applied to all specimens. The percentage area with positive color for each section was recorded.

\section{Analysis of Gene Expression by Real-Time Quantitative PCR}

Real-time PCR was performed as described before. ${ }^{16}$ RNA was prepared from the pooled samples using TRI Reagent (Sigma-Aldrich, St. Louis, MO). Complementary DNA was synthesized using reverse transcriptase from $200 \mathrm{ng}$ of total RNA according to the manufacturer's protocol (High Capacity cDNA Reverse Transcription Kit; Applied Biosystems, Foster City, CA). The following oligonucleotide primer pairs were used: IL- $1 \alpha$ sense, $5^{\prime}$ CAAACTGATGAAGCTCGTCA-3'; antisense, 5'-TCTCCTTGAGCGCTCACGAA-3'; IL-1 $\beta$ sense, 5'-TGGTGTGTGACGTTCCCATT-3'; antisense, 5'-CAGCACGAGGCTTTTTTGTTG-3'; MCP-1 sense, 5'-GCCCAGCACCAGCACCAG-3'; antisense, 5'-GGCATCACAGTCCGAGTCACAC-3'; MMP-9 sense, 5'-TGAGTGAGTTGGACTCTCGG-3'; antisense, 5' -CTCTCTACTGGGCGTTAGGG3'; TNF- $\alpha$ sense, 5'-TCCCAGGTTCTCTTCAAGGGA-3'; antisense, 5'-GGTGAGGAGCACGTAGTCGG-'; urokinase-type plasminogen activator (U-PA) sense, $5^{\prime}$-GCTCCTATAATCCTGGAGAGATGAA - 3'; antisense, 5'-ACCTGTCTTTTCAGCTTCTTCCCTCC-3'; 18S rRNA sense, 5'-AGTCGGAGGTTCGAAGACGAT-3'; antisense, 5'GCGGGTCATGGGAATAACG-3'. These primers were mixed with the Thunderbird SYBR qPCR Mix (Toyobo. Osaka, Japan). The ABI PRISM 7900HT machine (Applied Biosystems) was used for real-time PCR by denaturation at $95^{\circ} \mathrm{C}$ for 10 minutes, followed by 40 amplification cycles $\left(15\right.$ seconds at $95^{\circ} \mathrm{C}$ and 30 seconds at $60^{\circ} \mathrm{C}$ ). Each mRNA expression level was corrected by $18 \mathrm{~S}$ mRNA level. Data were analyzed using RQ Manager 1.2 (Applied Biosystems). 
Table 1. Characteristics of Wild-Type and $I L-1 R a^{-1-}$ Mice at 7 and 9 Months of Age

\begin{tabular}{|c|c|c|c|c|c|c|}
\hline & \multicolumn{3}{|c|}{7 months } & \multicolumn{3}{|c|}{9 months } \\
\hline & $\mathrm{WT}(n=6)$ & $R a^{-1-}(n=6)$ & $P$ value & $\mathrm{WT}(n=6)$ & $R a^{-1-}(n=6)$ & $P$ value \\
\hline BW (g) & $31.0 \pm 1.2$ & $27.1 \pm 0.8$ & 0.02 & $31.8 \pm 1.0$ & $27.5 \pm 0.7$ & 0.01 \\
\hline $\mathrm{SBP}(\mathrm{mm} \mathrm{Hg})$ & $92.2 \pm 3.9$ & $81.8 \pm 4.6$ & 0.12 & $96.3 \pm 8.3$ & $90.0 \pm 9.7$ & 0.63 \\
\hline T-chol (mg/dL) & $99.5 \pm 4.3$ & $86.5 \pm 4.4$ & 0.61 & $101.7 \pm 4.3$ & $101.5 \pm 3.9$ & 0.97 \\
\hline $\mathrm{HDL}(\mathrm{mg} / \mathrm{dL})$ & $79.5 \pm 5.5$ & $63.8 \pm 2.8$ & 0.66 & $61.8 \pm 3.7$ & $56.0 \pm 2.2$ & 0.21 \\
\hline $\mathrm{TG}(\mathrm{mg} / \mathrm{dL})$ & $40.6 \pm 2.0$ & $42.2 \pm 2.9$ & 0.67 & $50.3 \pm 4.1$ & $63.7 \pm 5.8$ & 0.09 \\
\hline Leukocytes & $41.5 \pm 2.6$ & $38.7 \pm 2.5$ & 0.44 & $41.0 \pm 1.9$ & $43.5 \pm 3.7$ & 0.56 \\
\hline Platelets & $90.0 \pm 3.8$ & $92.6 \pm 2.4$ & 0.58 & $83.6 \pm 6.7$ & $110.7 \pm 11.7$ & 0.07 \\
\hline
\end{tabular}

The results are shown as the mean \pm SEM

BW, body weight; HDL, high-density lipoprotein; SBP, systolic blood pressure; T-chol, total cholesterol; TG, triglyceride.

\section{Macrophages and T-Cell Activation Study}

T cells were prepared from the spleen and lymph nodes of $I L-1 R a^{-/-}$(6 to 8 week old) and WT (6 to 8 week old) mice, and then the cells were treated with hemolysis buffer $\left[17 \mathrm{mmol} / \mathrm{L}\right.$ Tris- $\mathrm{HCl}$ and $140 \mathrm{mmol} / \mathrm{L} \mathrm{NH}{ }_{4} \mathrm{Cl}(\mathrm{pH}$ 7.2)] to remove red blood cells, washed, and passed through a nylon wool column. Then anti-mouse B220 and anti-Mac-1 magnetic bead (Miltenyi Biotec, Bergisch Gladbach, Germany)-treated cells were passed through a MACS column (Miltenyi Biotec) to obtain T cells. Purified T cells $\left(2 \times 10^{5}\right.$ cells $)$ were plated on a 96-well plate coated with $10 \mu \mathrm{g} / \mathrm{mL}$ of anti-mouse CD3 mAb (145-2C11; BD Biosciences/Pharmingen) in a final volume of $200 \mu \mathrm{L}$ of Roswell Park Memorial Institute (RPMI) 1640 plus $10 \%$ fetal calf serum and cultured for 48 hours.

Peritoneal macrophages were recovered from mice 4 days after intraperitoneal injection of $2 \mathrm{~mL}$ of $2 \%$ thioglycollate broth (Nissui Pharmaceutical Co., Tokyo, Japan), and those $\left(2 \times 10^{5}\right.$ cells $)$ were plated on a 96-well plate and stimulated with $1 \mu \mathrm{g} / \mathrm{mL}$ lipopolysaccharide for 18 hours. Culture supernatant was then collected. We measured TNF- $\alpha$ levels in culture using enzyme-linked immunosorbent assay as described before. ${ }^{18}$

\section{In Vitro Migration Assay}

Migration of mouse peritoneal macrophages was measured in a modified Boyden chamber migration assay using the Chemicon QCM 96-well (5 $\mu \mathrm{m}$ ) Cell Migration Assay kit (Millipore, Billerica, MA). A total of $1 \times 10^{5}$ cells from WT or IL-1Ra ${ }^{-/-}$mice were loaded into the migration chamber, and medium containing $10 \mathrm{ng} / \mathrm{mL}$ MCP-1 was placed in the lower chamber. After allowing cell migration for 12 hours, cells were removed from the upper side of the membrane, and migratory cells on the lower side of the membrane were collected. These cells were lysed and detected by the patented CyQuant GR dye.

\section{Bone Marrow Cell Transplantation}

To elucidate the role of bone marrow (BM) cells in the development of femoral artery aneurysms, BM cell transplantation (BMT) was performed as described previ- ously. ${ }^{18}$ For BM cell transplantation, BM cells were taken from femurs, tibias, and pelvises of $I L-1 R a^{-1-}$ ( 5 to 6 week old) and WT (5 to 6 week old) mice and were treated with hemolysis buffer. Purified BM cells ( $10^{7}$ cells/ mouse) in $0.2 \mathrm{~mL}$ of phosphate-buffered saline (PBS) were transplanted intravenously into lethally irradiated (750 rad) recipient mice at 6 weeks of age.

\section{Statistical Analysis}

The results are shown as mean \pm SEM. Statistical analysis was performed by unpaired $t$-test or analysis of variance. A value of $P<0.05$ was regarded as a significant difference. All tests were performed using SPSS software, version 14.0 (IBM, Armonk, NY).

\section{Results}

\section{IL-1 $\mathrm{Ra}^{-1-}$ Mice Spontaneously Develop Fusiform Femoral Artery Aneurysms}

Phenotypic analysis of $I L-1 R a^{-1-}$ mice backcrossed to the C57BL/6J background for eight generations revealed that the body weight of 7 -month-old $I L-1 R a^{-/-}$male mice was lower than that of the WT mice ( $n=6$ for each group) (Table 1). We observed no significant differences in systolic blood pressure, plasma total cholesterol levels, or high-density lipoprotein cholesterol levels, triglyceride levels, leukocyte counts, or platelet counts between groups ( $n=6$ for each group) (Table 1). In addition, femoral artery diameter was similar in $I L-1 R^{-/-}(n=12)$ and WT mice $(n=12)$. However, $I L-1 R a^{-\prime-}$ mice $(n=12)$ spontaneously developed fusiform femoral artery aneurysms (Figure 1A) after 8 months of age, and both maximal vessel area and intimal + medial area of femoral arteries in such mice were significantly larger compared with WT mice $(n=12)$ after 8 months of age (Figure 1B). We observed numerous inflammatory cells around the fusiform femoral artery aneurysm in $I L-1 R a^{-1-}$ mice, and the lumen was larger in $I L-1 R a^{-/-}$mice compared with WT mice (Figure 1A). The maximal vessel area and intima + medial area of aneurysms in 9-month-old $I L-1 R a^{-/-}$mice $(n=12)$ were significantly larger compared with 8-month-old IL-1Ra ${ }^{-1-}$ mice (Figure 1B). No WT mice developed femoral artery aneurysms during our experimental period (Figure 1B). We checked characteristics in 

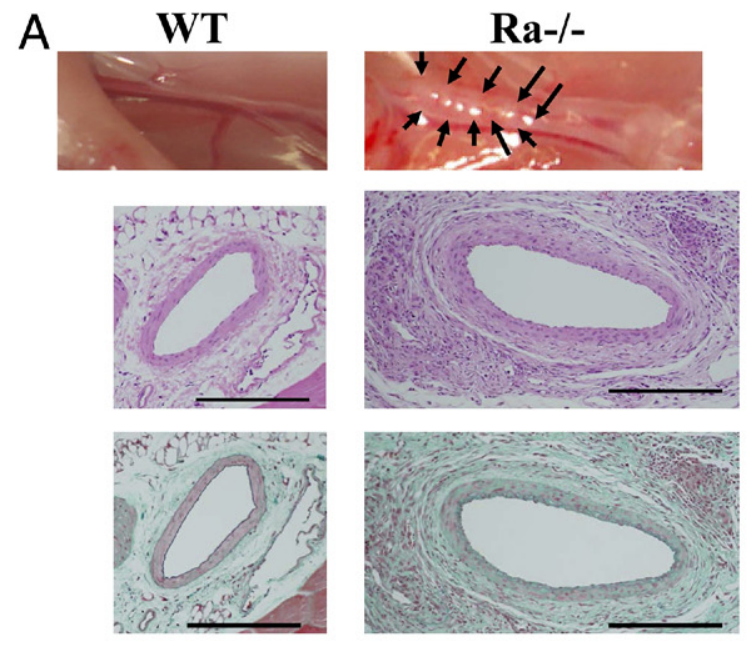

$\mathrm{B}$
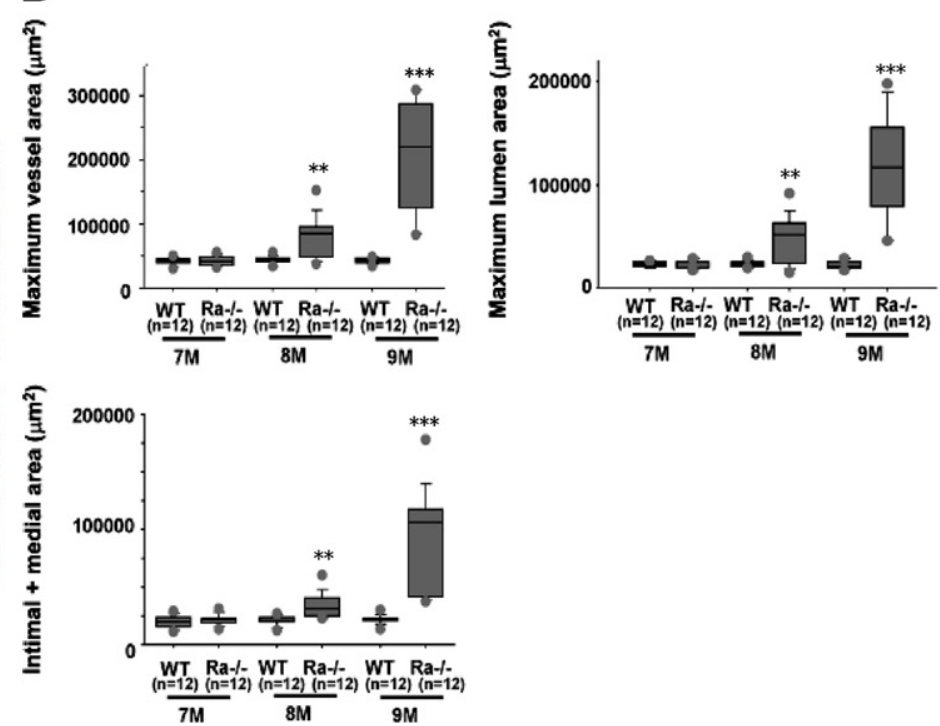

Figure 1. $I L-1 R a^{-/-}$mice spontaneously developed femoral artery aneurysms. A: Macroscopic appearance of femoral arteries of $I L-1 R a^{-1-}$ (right panel) and WT mice (left panel) at 8 months of age (upper panels). Femoral artery sections were stained with hematoxylin and eosin (middle panels) or Masson's trichrome (lower panels). Scale bar $=200 \mu \mathrm{m}$. B: Femoral artery specimens were taken from $I L-1 R a^{-1-}$ and WT mice at 7 months $(n=12$ for each group), 8 months ( $n=12$ for each group), or 9 months $\left(n=12\right.$ for each group). We analyzed maximal vessel area (left) as well as intima + medial area (right). ${ }^{* *} P<$ $0.01,{ }^{* * * * *} P<0.001$.

both groups at 9 months of age ( $n=6$ for each group) (Table 1). Triglyceride levels and platelet counts tended to increased in $I L-1 R a^{-/-}$mice compared to WT mice, but did not reached statistical significance (Table 1).

We performed histological examination of the maximal size of femoral arteries from $I L-1 R a^{-/-}$mice at $7,8,9$, and 10 months of age (Figure 2A). At 7 months of age, the femoral artery media consisted predominantly of SMCs (brown layer) and few connective tissues (red fibers), and lacked intimal hyperplasia. By contrast, 8-month-old IL$1 R a^{-1-}$ mice showed increased medial thickness and elevated SMCs. Furthermore, the aneurismal arterial wall of 9-month-old IL-1Ra ${ }^{-/-}$mice developed intimal hyperplasia, resulting in more pronounced thickness. Moreover, elastin staining revealed destruction of the internal and external elastic lamina. After 10 months of age, the intimal + medial area of aneurismal femoral arteries plateaued in $I L-1 R a^{-1-}$ mice, and histological analysis showed acellular layers in the media of aneurismal femoral arteries (Figure 2A). In contrast, nonaneurismal femoral arteries in $I L-1 R a^{-1-}$ mice retained their normal structures, and we observed no inflammatory cells in the adventitia (Figure 2A). At 10 months, immunostaining revealed that the $\alpha$-SMA-positive area in the media of $I L$ $1 R a^{-1-}$ mice $(n=6)$ was significantly decreased compared with WT mice $(n=6)(P<0.0001)$ (Figure 2B). Importantly, at 9 months, the cleaved caspase-3-positive apoptotic index was significantly increased in the media of IL-1Ra ${ }^{-1-}$ mice $(n=6)$ compared with that of WT mice $(n=6)(P<0.0001)$ (Figure 2C).

The longitudinal sections displayed the entire developmental progression of aneurysms from normal tissue to aneurism formation (Figure 3B). We observed many inflammatory cells in the adventitia at the initiation site of neointima formation, and also detected MMP-9 protein expression in the media and neointima, and several inflammatory cells at the initiation site (Figure 3B). As shown Figure 3C, the percent positive area of MMP-9 in $I L-1 R a^{-/-}$mice $(n=6)$ was much larger than in WT mice $(n=6)$. Furthermore, we observed TNF- $\alpha$ protein expression in neointimal cells and in the many inflammatory cells found in the adventitia at the site, and quantitative analysis also revealed TNF- $\alpha$ expression increased significantly in 9-month-old $\mathrm{IL-1Ra^{-1- }}(n=6)$ compared to 9-month-old WT mice $(n=6)$ (Figure 3, B and C). These changes (ie, accumulation of inflammatory cells in the adventitia, and expression of TNF- $\alpha$ and MMP-9) might contribute to aneurysm formation. Indeed, the changes did not occur in the normal femoral arteries of WT mice (Figure 3A). The normal femoral artery is a muscular artery, consisting mainly of SMCs in the media and little collagen (Figure 3A). Many collagens were detected, mainly in the adventitia. By contrast, we detected collagen in the neointima of femoral artery aneurysm, and polarization microscopy showed the collagen present as a green color (Figure 3B).

The detection rates of both bilateral femoral artery aneurysms $(16.6 \%$ at 8 months, $50 \%$ at 9 months, and $58.2 \%$ at 10 months) and multiple aneurysms with other peripheral artery aneurysms (ie, deep femoral artery aneurysm: $0 \%$ at 8 months, $33.3 \%$ at 9 months, and $33.3 \%$ at 10 months; popliteal artery aneurysm: $0 \%$ at 8 months, 33.3\% at 9 months, and 50\% at 10 months) increased with age. Interestingly, longitudinal sections

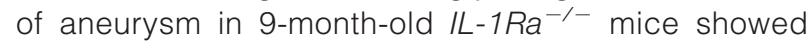
many inflammatory cells, predominantly in the adventitia at the aneurysm initiation site (Figure 4). Immunostaining revealed that the majority of inflammatory cells were granulocytes. Moreover, we detected several 


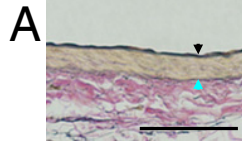

$7 \mathrm{M}$

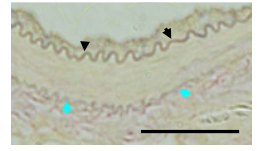

$8 \mathrm{M}$

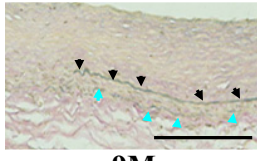

9M

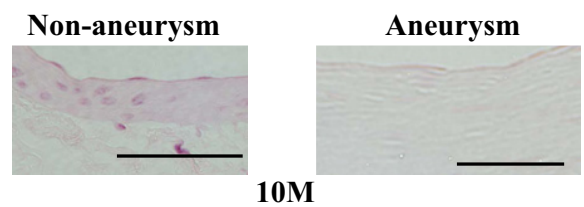

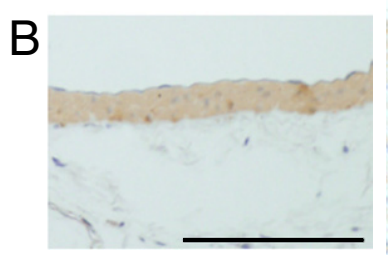

WT (10M)

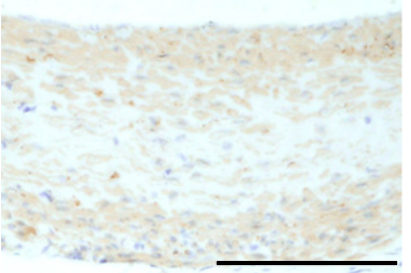

Ra-/- (10M)

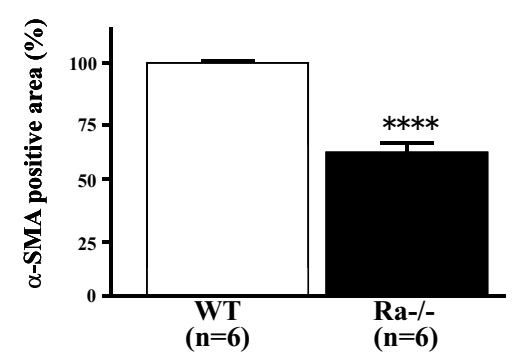

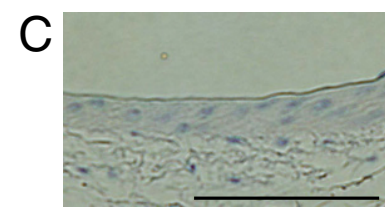

WT (9M)

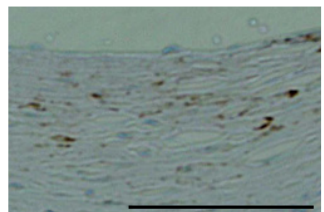

Ra-/- (9M)

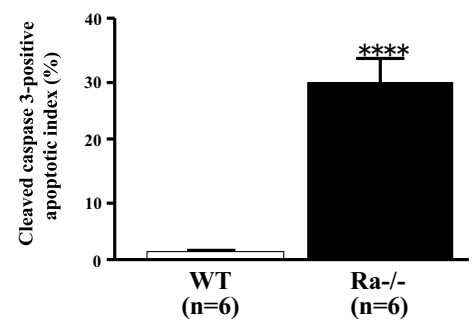

Figure 2. Time-dependent changes of histological findings of fusiform femoral artery aneurysms. A: Histological sections of femoral arteries of $I L-1 R a^{-1-}$ mice at 7 months (upper left), 8 months ( $8 \mathrm{M}$, upper middle), 9 months (9M, upper right), and 10 months (10M, lower panels). The sections were stained with elastica van Gieson (upper panels) or hematoxylin and eosin (lower panels). Scale bar $=100 \mu \mathrm{m}$. Black arrows show the internal elastic lamina, and blue arrows show the external elastic lamina. B: Microscopic appearance for $\alpha$-SMA staining of femoral arteries from WT (upper left) and $I L-1 R a^{-/}$mice (upper right) at 10 months $(10 \mathrm{M})$ of age. Scale bar $=100 \mu \mathrm{m}$. Lower graph shows quantification of $\alpha$-SMA-positive area in the media of 10 -month-old $I L-1 R a^{-/-}$ $(n=6)$ and WT mice $(n=6) .{ }^{* * * * *} P<0.0001$. C: Microscopic appearance for cleaved caspase-3 staining of femoral arteries from WT (upper left) and $I L-1 R a^{-/-}$mice (upper right) at 9 months $(9 \mathrm{M})$ of age. Scale bar $=$ $100 \mu \mathrm{m}$. Lower: graph shows cleaved caspase-3-positive apoptotic indexes in the media of 9-month-old $I L-1 R a^{-/-}(n=6)$ and WT mice $(n=$ 6). ${ }^{* * * * *} P<0.0001$ macrophages (Figure 4) and a few T cells (data not shown) at the same site.

\section{Enhanced mRNA Expression of Cytokines, Chemokines, and the Regulation Genes of Extracellular Matrix in Femoral Aneurysms in IL-1Ra ${ }^{-1-}$ Mice}

Using femoral arteries of 7- and 9-month-old WT ( $n=6$ for each group) and 9-month-old IL-1Ra ${ }^{-/-}$mice ( $n=6$ for each group), we analyzed mRNA expression of genes involved in cytokines and chemokines, and the selected genes that regulate extracellular matrix formation. Notably, we used the sites of femoral artery aneurysms and nonaneurismal arteries to analyze the arteries of 9-monthold IL-1Ra ${ }^{-/-}$mice, and normal arteries of 9-month-old WT mice. mRNA levels did not differ significantly between WT and IL-1Ra ${ }^{-\prime-}$ mice before aneurysm formation (at 7 months of age) (data not shown). By contrast, real-time PCR of aneurysms in 9-month-old $I L-1 R a^{-/-}$mice revealed significantly increased mRNA levels of IL-1 $\alpha$ (6.4and 3.8-fold, $P<0.05$ ), IL-1 $\beta$ (7.0- and 4.1-fold, $P<$ $0.05)$ and TNF- $\alpha$ (13.3- and 8.9-fold, $P<0.05)$ compared with arteries in WT mice and nonaneurysm arteries in IL-1Ra ${ }^{-/-}$mice (Figure 5). Furthermore, MCP-1 mRNA levels increased significantly in aneurysms in $I L-1 R a^{-1-}$ mice (8.2- and 5.4-fold, $P<0.05$ ) compared with normal arteries in both WT and IL-1Ra ${ }^{-1-}$ mice (Figure 5). In the selected genes regulating extracellular matrix formation, mRNA levels of u-PA (7.5- and 5.6-fold, $P<0.05)$ and MMP-9 (6.0- and 4.7-fold, $P<0.01$ ) were significantly higher in aneurysms of $I L-1 R a^{-/-}$mice compared with normal arteries in both WT and IL-1Ra ${ }^{-/-}$mice (Figure 5). Therefore, IL-1Ra deficiency may induce chronic medial and adventitial inflammation and aneurysm development, possibly due to enhanced mRNA expression of cytokines and chemokines, and the regulation genes of extracellular matrix. We also investigated mRNA expression in the ascending aorta, aortic arch, descending aorta, and abdominal aorta of both groups. Only MMP-9 mRNA levels in the abdominal aorta in $/ L-1 R a^{-/-}$mice were significantly higher than those in WT mice $(P<0.05)$; other genes did not differ significantly between groups. Very few IL-1Ra ${ }^{-1-}$ mice $(2.4 \%, 2$ of 83 ), and no WT mice developed abdominal aortic aneurysms. Neither group developed thoracic aortic aneurysms. Our results suggest that $I L-1 R a^{-1-}$ mice changed mRNA expression and developed aneurysm in femoral artery specifically.

\section{Spontaneous Development of Saccular, Dissecting, and Thrombosed Large Femoral Artery Aneurysms in IL-1Ra ${ }^{-1-}$ Mice}

Interestingly, we frequently observed saccular aneurysms, which appear like a small blister on the side of the femoral artery (Figure 6, A and B), with fusiform aneurysms in 10 -month-old $I L-1 R a^{-1-}$ mice $(75 \%, 9$ of 12$)$. The detection rate for saccular aneurysms on the femoral 
A
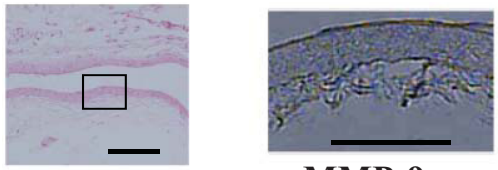

MMP-9
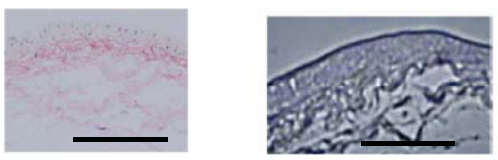

TNF- $\alpha$

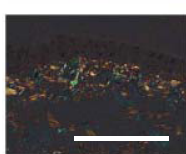

Sirius Red

C

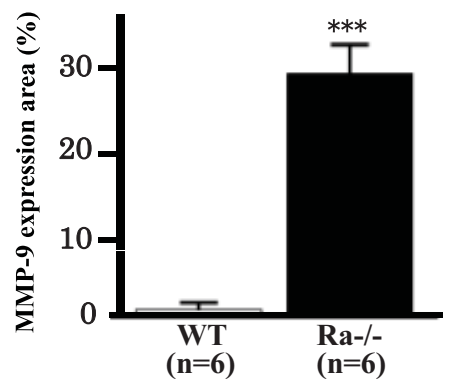

B
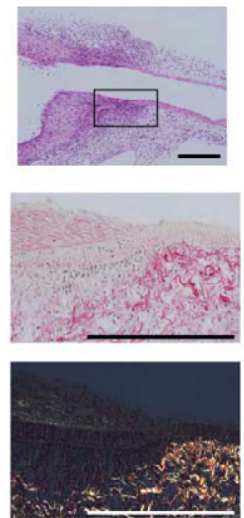

Sirius Red

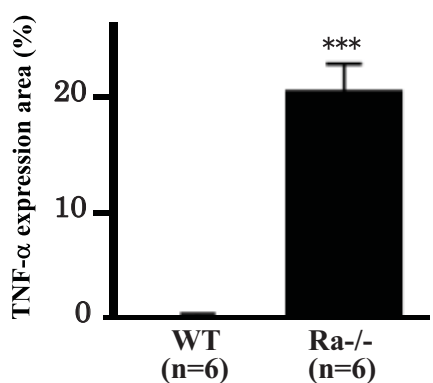

Figure 3. Histological findings of fusiform femoral artery aneurysms. A: The longitudinal sample from a 9-month-old WT mouse was stained with hematoxylin and eosin (upper left) Boxed area is shown below and in right panels. Middle left panel shows Picrosirius Red staining without polarization. Lower left pane showed Picrosirius Red staining viewed under linearity polarized light in the same section as in the upper panel. The serial sections were stained with MMP-9 (upper right) or TNF- $\alpha$ (lower right). Scale bar $=100 \mu \mathrm{m}$. B: The longitudinal femoral artery aneurysm sample from a 9-month-old $I L-1 R a^{-1}$ mouse was stained with hematoxylin and eosin (upper left). Boxed area is shown below and in right panels. Middle left panel shows Picrosirius Red staining without polarization. Lower left panel showed Picrosirius Red staining viewed under linearity polarized light in the same section as in the upper panel. The serial sections were stained for MMP-9 (upper right) or TNF- $\alpha$ (lower right). Scale bar $=200 \mu \mathrm{m}$. C: Quantification of MMP-9 and TNF- $\alpha$ expression in the femoral arteries of 9-month-old $I L-1 R a^{-1-}(n=$ 6) and WT mice $(n=6)$. ${ }^{* * * * *} P<0.001$. artery increased with age $(0 \%$ at 8 months, $25 \%$ at 9 months, and $75 \%$ at 10 months).

Although the femoral artery aneurysms of several 9-month-old $\mathrm{IL}-1 \mathrm{Ra}^{-1-}$ mice $(6.4 \%, 6$ of 93) were red (Figure 6C), the majority were white (Figure 1A). Following perfusion, we harvested, fixed, and sectioned the aneurysms that showed as red. Remarkably, intimal and medial thickening increased in the aneurysms that showed red, and dissection occurred between the intima and media (Figure 6D). Elastin staining revealed frag-
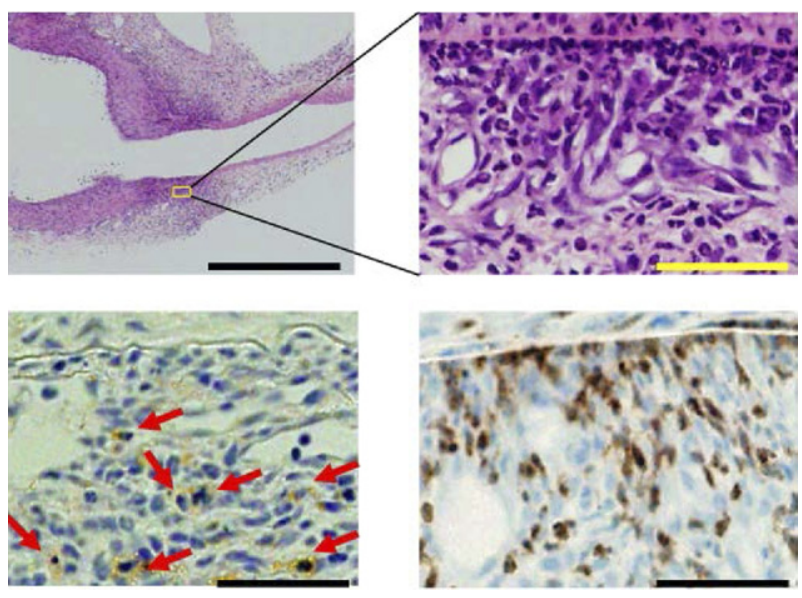

Figure 4. Immunostaining findings of fusiform aneurysms. The longitudinal sample from a 9-month-old $I L-1 R a^{-/-}$mouse was stained with hematoxylin and eosin (upper left). Scale bar $=500 \mu \mathrm{m}$. Boxed area is shown in upper right panel. Scale bar $=50 \mu \mathrm{m}$. Immunostaining for macrophages (lower left) and granulocytes (lower right) was performed. Scale bar $=50 \mu \mathrm{m}$. Red arrows indicate postive cells (macrophages). mentation of the internal and external elastic lamina (Figure $6 \mathrm{D}$ ) in $/ L-1 R a^{-/-}$mice.

Furthermore, several thrombosed large aneurysms of femoral and/or popliteal arteries were detected in 10month-old IL-1Ra-'- mice (24\%, 7 of 29) (Figure 6E). However, we did not find any ruptured large aneurysms.

\section{Activation of IL-1Ra ${ }^{-1-}$ Macrophages and $T$ Cells Is Excessive Compared with WT Mice}

We next studied macrophage and T-cell function. Following lipopolysaccharide $(1 \mu \mathrm{g} / \mathrm{mL})$ stimulation, IL-1Ra-de-

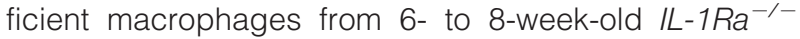
mice produced much higher levels of TNF- $\alpha$ compared with 6- to 8-week-old WT mice $(n=6)$ (Figure 7A). Furthermore, TNF- $\alpha$ production of T cells in 6- to 8-week-old IL-1Ra ${ }^{-1-}$ mice stimulated with anti-CD3 antibody was higher compared with 6- to 8-week-old WT mice $(n=6)$ (Figure 7A). Our results suggest excessive activation of IL-1Ra-deficient macrophages and $\mathrm{T}$ cells compared with WT mice, even at the same levels of stimulation.

\section{Migration Activity of $\mathrm{IL}-1 \mathrm{Ra}^{-1-}$ Macrophages is Increased Compared with that of WT Macrophages}

As shown in Table 1, total counts of leukocytes were similar between WT and $\mathrm{L}-1 \mathrm{Ra}^{-1-}$ mice at both 7 and 9 months of age, suggesting proliferation of peripheral leukocytes were not increased in $/ \mathrm{L}-1 \mathrm{Ra}^{-/-}$mice compared 

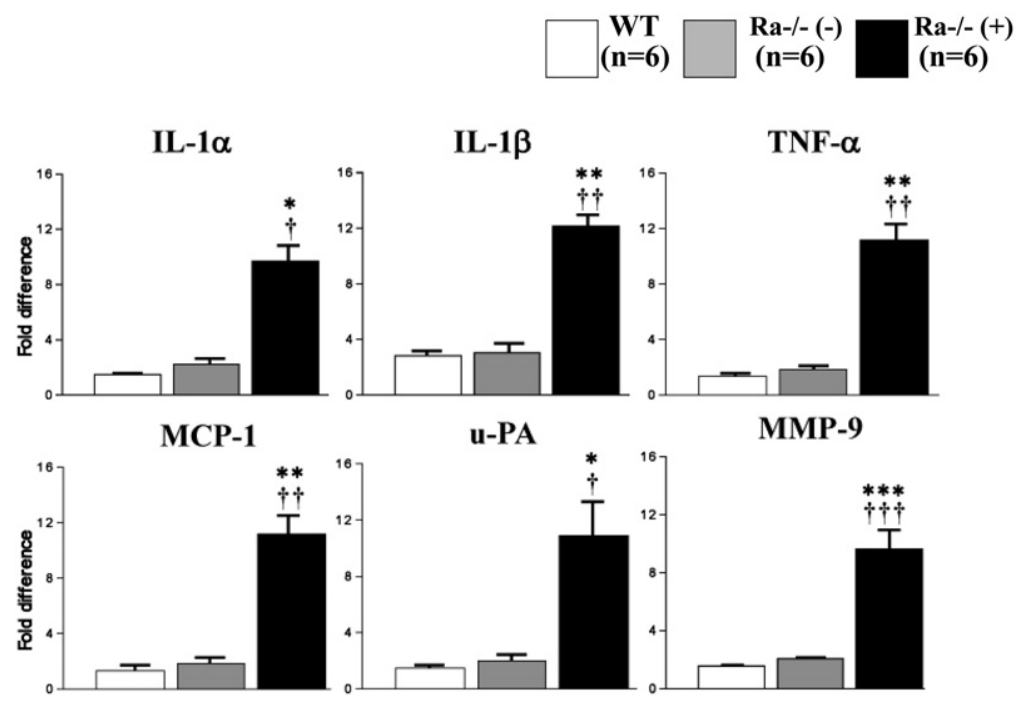

Figure 5. Enhanced mRNA expression of cytokines, chemokines, and the regulation genes of extracellular matrix in femoral aneurysms in $\mathrm{IL}-1 \mathrm{Ra}^{-/-}$mice. mRNA levels of the indicated genes in the femoral arteries of 9-month old of WT ( $n=$ $6)$, nonaneurismal artery in $I L-1 \mathrm{Ra}^{-/-}(n=6)$, and aneurisma artery in $I L-1 R a^{-1-}$ mice $(n=6)$ were quantified using realtime PCR. Degree of change in gene expression is based on the 7 -month baseline expression level of WT mice. ${ }^{*} P<0.05$ WT versus $R a^{-/-}(+) ;{ }^{* * *} P<0.01$ WT versus $R a^{-/-}(+) ;{ }^{* * * *} P<0.001$ WT versus $R a^{-/-}(+) ;{ }^{\dagger} P<0.05 R a^{-/-}(-)$versus $R a^{-}$ $-(+)$; ${ }^{\text {t}} P<0.01 R^{-/-}(-)$versus $R a^{-/-}(+)$; ${ }^{\text {t十 }} P<0.001$ $\mathrm{Ra}^{-/-}(-)$versus $\mathrm{Ra}^{-/-}(+)$.

with WT mice. Therefore, further experiments determined the chemotactic response of WT and $I L-1 R a^{-/-}$macrophages to MCP-1. The migration activity of $I L-1 R a^{-/-}$ macrophages ( $n=6$ ) were 4.2-fold higher than that of WT macrophages ( $n=6$ ) (Figure 7B). These findings indicated that the increased presence of inflammatory cells in lesions of $\mathrm{L}-1 \mathrm{Ra}^{-/-}$mice could result from increased recruitment of inflammatory cells.
A

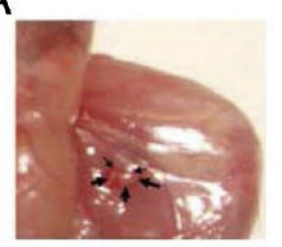

C

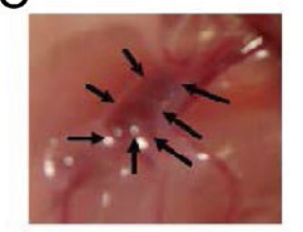

$E$

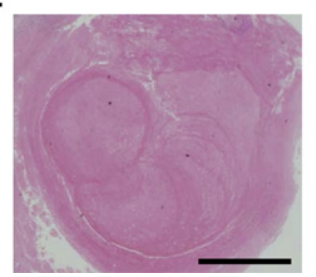

B

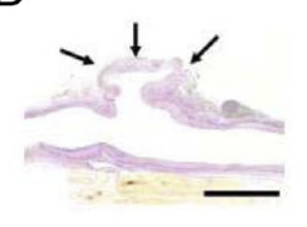

D

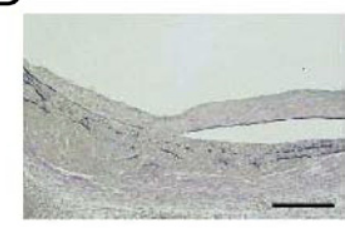

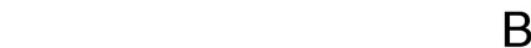

B
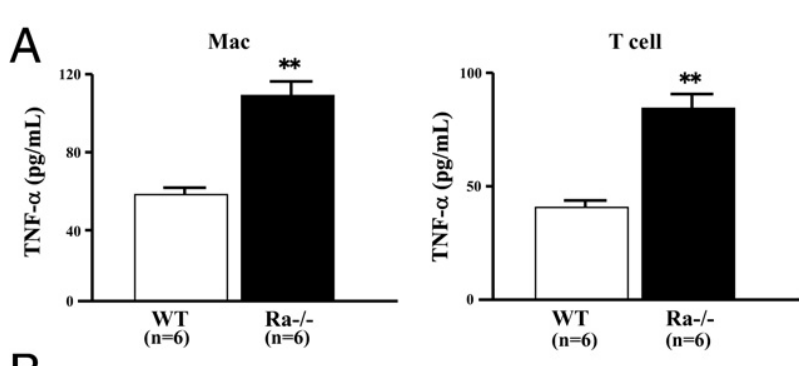

IL-1Ra Deficiency in Hematopoietic Cell Types

Participates Crucially in the Development of Femoral Artery Aneurysm

To test the hypothesis that IL-1Ra deficiency in hematopoietic cell types contributes importantly to the development of femoral artery aneurysm, we performed BMT. After injecting BM from $I L-1 R a^{-/-}$or WT mice into lethally irradiated WT mice, we detected femoral artery aneurysms with increased intimal + medial area and many inflammatory cells in the adventitia of several WT mice (55\%, 6 of 11$)$ that received $\mathrm{BM}$ cells from $/ L-1 R a^{-1-}$ mice, but not from WT mice (0\%, 0 of 10) (Figure $8 \mathrm{~A}$ ) at 8 months post-BMT. Quantitative analyses also showed

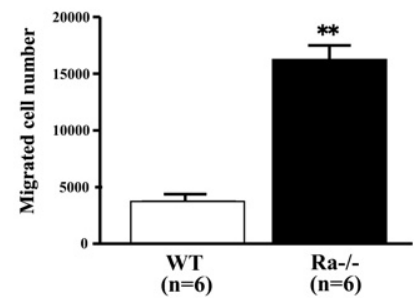

Figure 6. Histological findings of saccular dissecting and thrombosed femoral artery aneurysms in $I L-1 R a^{-/-}$mice. A: The macroscopic appearance of the femoral artery showed saccular aneurysm (black arrows) in a 10-monthold $I L-1 R a^{-/-}$mouse. B: The longitudinal section of saccular aneurysm in a 10-month-old $I L-1 R a^{-1-}$ mouse was stained with elastica van Gieson. Scale bar $=1 \mathrm{~mm}$. C: The macroscopic appearance of the femoral artery showed as red (black arrows) in a 9-month-old $I L-1 \mathrm{Ra}^{-1-}$ mouse. D: The longitudinal sample from a 9-month-old $I L-1 R a^{-1-}$ mouse was stained with elastica van Gieson (scale bar $=200 \mu \mathrm{m}$ ). E: The cross section of a thrombosed large aneurysm in a 10 -month-old $I L-1 R a^{-/-}$mouse was stained with hematoxylin and eosin. Scale bar $=2 \mathrm{~mm}$. 

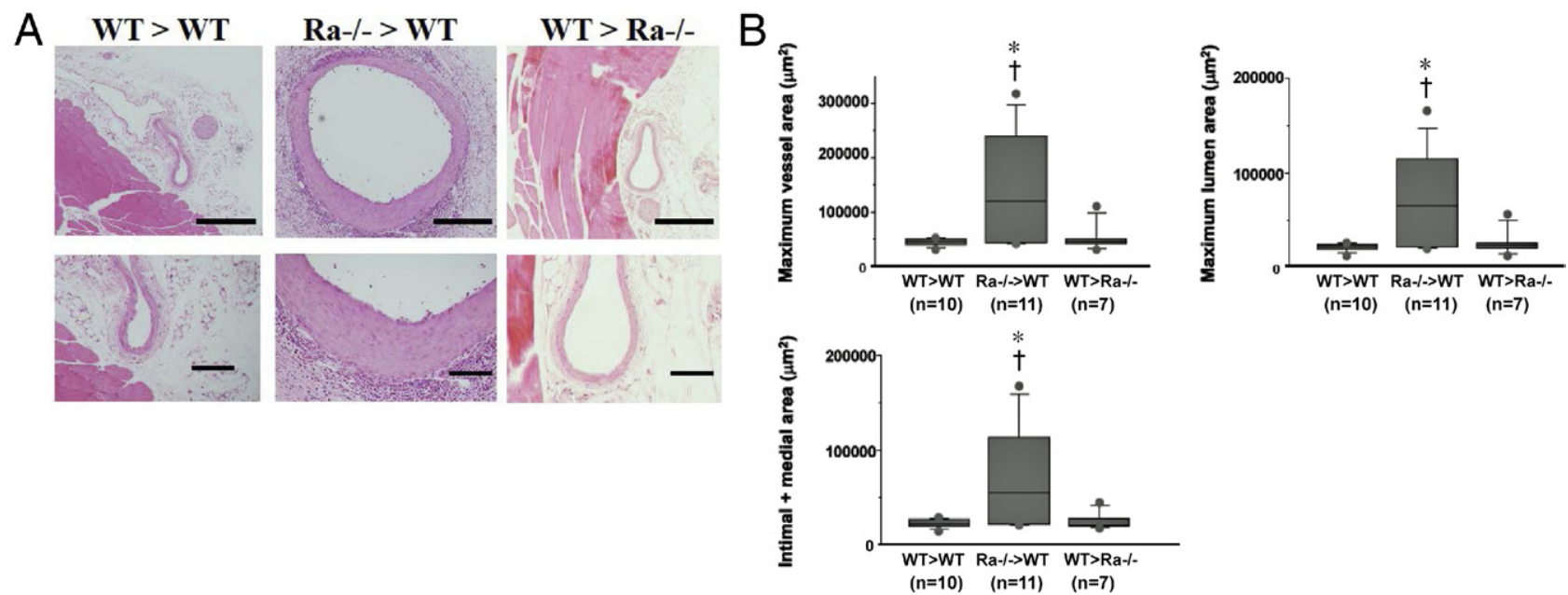

Figure 8. IL-1Ra deficiency in hematopoietic cell types participates in the development of femoral artery aneurysm. A: Bone marrow (BM) of $I L-1 R a^{-/-}$( middle $\mathrm{Ra}^{-/-}>$WT) or WT (left, WT $>$WT) mice was injected in lethally irradiated recipient WT mice. Furthermore, BM of WT mice was injected in lethally irradiated recipient $I L-1 R a^{-1-}$ mice (right, WT $>\mathrm{Ra}^{-1-}$ ). We detected femoral artery aneurysms in several WT mice receiving BM cells from $I L-1 R a^{-\prime-}$ mice [upper middle $($ scale bar $=500 \mu \mathrm{m})$ and lower middle $($ scale bar $=200 \mu \mathrm{m})]$, but not WT mice [upper left $(\mathrm{scale}$ bar $=500 \mu \mathrm{m})$ and lower left $(\mathrm{scale}$ bar $=200 \mu \mathrm{m})$ ]. Moreover, BM of WT mice significantly suppressed the development of femoral artery aneurysms in $I L-1 R a^{-/}$mice $[\mathbf{u p p e r}$ right $(\mathrm{scale}$ bar $=500 \mu \mathrm{m})$ and lower right $($ scale bar $=200 \mu \mathrm{m})]$. $(\mathbf{B})$ Femoral artery specimens were taken from WT mice receiving BM cells from $I L-1 R a^{-/-}(n=11)$ or WT mice $(n=10)$, and $I L-1 R a^{-1-}$ mice receiving BM cells from WT mice $(n=7)$ at 8 months after bone marrow cell transplantation. We analyzed maximal vessel area (upper left), maximal lumen area (upper right), and intima + medial area (lower left) ${ }^{*} P<0.05 R a^{-/-}>$WT versus WT $>$WT; ${ }^{\dagger} P<0.05 R a^{-/-}>$WT versus WT $>R a^{-/-}$.

maximal vessel area, maximal lumen area, and intima + medial area were significantly larger in WT mice receiving BM cells from IL-1Ra ${ }^{-/-}$compared with WT mice receiving BM cells from WT mice at 8 months post-BMT (Figure 8B). In contrast, BM of WT mice significantly suppressed the development of femoral artery aneurysm in $/ \mathrm{L}-1 \mathrm{Ra}^{-1-}$ mice at 8 months post-BMT (14\%, 1/7) (Figure 8A), and quantitative analyses revealed maximal vessel area, maximal lumen area, and intima + medial area were significantly smaller in $/ L-1 R a^{-/-}$mice receiving BM cells from WT mice compared with WT mice receiving BM cells from $\mathrm{L}-1 \mathrm{Ra}^{-1-}$ mice at 8 months post-BMT (Figure $8 \mathrm{~B}$ ). Thus, IL-1Ra-deficient hematopoietic cells induce femoral artery aneurysm.

\section{Discussion}

Femoral artery aneurysms are rare, and their natural history is poorly understood. Occurring most frequently in elderly persons, ${ }^{8,9}$ such aneurysms are often bilateral and may associate with multiple aneurysms. A previous report showed that $36 \%$ of patients with a femoral artery aneurysm also had a contralateral femoral artery aneurysm, and $69 \%$ had multiple aneurysms. ${ }^{10}$ Our study detected femoral artery aneurysms in $I L-1 R a^{-1-}$ mice older than 8 months of age. Among 10-month-old IL$1 R a^{-1-}$ mice, $58 \%$ (7 of 12) had bilateral femoral artery aneurysms and $50 \%$ (6 of 12) had popliteal artery aneurysms. These findings are very similar to results previously reported in humans. ${ }^{8-10}$

Yamamoto et $\mathrm{al}^{9}$ reported that femoral artery aneurysms with a background of arteriomegaly defined as diffuse arterial enlargement involving several arterial segments, might represent a subgroup of patients at risk for multiple femoral aneurysms. In the results reported here, $67 \%$ (8 of 12) of 8 -month-old $/ L-1 R a^{-1-}$ mice demon- strated diffuse long fusiform femoral artery aneurysms; almost all mice with such lesions developed multiple aneurysms. Thus, if diffuse long fusiform lesions were described as arteriomegaly, our results might support Yamamoto and colleagues' suggestions. Interestingly, such dilating diathesis has not been observed in patients with abdominal aortic aneurysm (AAA). ${ }^{11}$ In our study, very few IL-1Ra ${ }^{-1-}$ mice developed AAA (2.4\%, 2 of 83 ), suggesting that other specific factors-genetic or other-may participate in the development of femoral artery aneurysm.

Aneurysms in peripheral arteries may associate with etiological factors such as infectious arteritis, noninfectious immunological or inflammatory arteritis, and connective tissue diseases, but atherosclerotic aneurysms occur frequently. ${ }^{12,13}$ However, the notion that atherosclerotic changes in the aneurismal wall cause or result from arterial aneurysm remains controversial. The current study showed no significant differences in plasma total cholesterol levels, triglyceride levels, or high-density lipoprotein cholesterol levels between $I L-1 R a^{-1-}$ and WT mice at 7 and 9 months of age. Interestingly, a comparison between 7-month-old and 9-month-old mice in the same strain revealed that increased total cholesterol levels $(P=0.03)$ with an increase in the levels of triglyceride $(P=0.007)$ were detected in $I L-1 R a^{-1-}$ mice, but were not significant in WT mice. Increased triglyceride levels might induce the development of aneurysm, but we thought that chronic inflammation in $I L-1 R a^{-1-}$ mice should increase triglyceride levels. Furthermore, we detected no atherosclerotic occlusive lesions in arteries. Our results suggest that femoral artery aneurysm may associate mainly with inflammation, not atherosclerotic changes. However, atherosclerosis involves an ongoing inflammatory response, ${ }^{19}$ and we believe atherosclero- 
sis-induced inflammation likely contributes to femoral artery aneurysm.

The normal femoral artery is a muscular artery, characterized mainly by SMCs in the media and little collagen. In contrast, Sirius Red staining revealed collagen in the neointima of femoral artery aneurysm, and polarization microscopy showed the collagen presented as green, suggesting several collagen type IIIs might be included in it. ${ }^{20}$ These findings might be supported by a previous report that showed collagen, especially type III, synthesis increases during the early stages of aneurysm formation. ${ }^{21}$ Furthermore, we found high collagen content at the initiation site of aneurysm formation. In early stages of aneurysm, interstitial collagen in the adventitia provides tensile strength to the arterial wall. In later stages, collagen degeneration exceeds its synthesis, ultimately favoring the enlargement of aneurysm. ${ }^{22}$ We also detected several elastic laminas at the initiation site of aneurysm formation (data not shown). By contrast, we observed only two elastic laminas (internal and external) at the normal site. However, elastic lamina degradation exceeds its synthesis in later stages of aneurysm formation (accompanied by excessive degradation of other extracellular matrix macromolecules), ultimately favoring arterial wall dissection. Increased turnover and loss of elastin fibers caused by increased MMP expression probably underlie femoral artery dilation and dissection.

We detected that TNF- $\alpha$ protein expression was significantly higher in the femoral arteries in $I L-1 R a^{-/-}$mice compared to WT mice. A previous report showed that TNF- $\alpha$ can initiate apoptosis in many cell types. ${ }^{23}$ Indeed, immunostaining revealed that the cleaved caspase-3positive apoptotic index was significantly increased in the media of $I L-1 R a^{-1-}$ mice compared with that of WT mice at 9 months of age $(P<0.0001)$. Furthermore, the $\alpha$-SMA-positive area in the media of $I L-1 \mathrm{Ra}^{-1-}$ mice was significantly decreased compared with WT mice $(P<$ $0.0001)$. These processes induced by excessive inflammation in $I L-1 R a^{-/-}$mice should act in concert to progressively weaken the arterial wall, resulting in dilation and aneurysm formation.

Real-time PCR of aneurysms in 9-month-old $/ \mathrm{L}-1 \mathrm{Ra}^{-/-}$ mice revealed significantly increased mRNA levels of cytokines and chemokines, and the regulation genes of extracellular matrix compared with both normal arteries in WT mice and nonaneurismal arteries in $I L-1 R a^{-1-}$ mice. Histological analysis showed that nonaneurismal femoral arteries in $I L-1 R a^{-1-}$ mice retained normal structures without any inflammatory cells in the adventitia. These data strengthen the results of real-time PCR, suggesting that altered mRNA might be a specific change for aneurysm formation. Taken together, increased femoral artery aneurysm formation in $/ \mathrm{L}-1 \mathrm{Ra}^{-/-}$mice results from greater local inflammation induced by cytokines, chemokines, and MMP.

IL-1Ra-deficient macrophages and $\mathrm{T}$ cells produced much higher levels of TNF- $\alpha$ compared to WT mice receiving the same stimulation. Our findings suggest that excessive activation occurs more frequently in IL-1Radeficient macrophages and $T$ cells compared with those from WT mice. Furthermore, we also showed that the migration activity of $\mathrm{L}-1 \mathrm{Ra}^{-1-}$ macrophages were 4.2fold higher than that of WT macrophages. Taken together, $\mathrm{L}-1 \mathrm{Ra}^{-1-}$ inflammatory cells increased migration activity and production of proinflammatory cytokines around the femoral artery, thus inducing inflammatory femoral artery aneurysm.

Because our results also suggested that IL-1Ra deficiency in inflammatory cells might promote aneurysms, we performed BMT. We observed femoral artery aneurysms in several WT mice receiving BM cells from $I L$ $1 R a^{-1-}$ mice $(55 \%)$, but not WT mice $(0 \%)$, demonstrating that IL-1Ra deficiency in inflammatory cells induces aneurysm. IL-1Ra protein expressed by endothelial cells ${ }^{15}$ and $\mathrm{SMCs}^{24}$ may protect the vascular wall from inflammation induced by IL-1Ra-deficient inflammatory cells, possibly explaining why only $55 \%$ of WT mice receiving IL-1Ra-deficient BM cells developed femoral artery aneurysms. In contrast, BM of WT mice significantly suppressed the development of femoral artery aneurysm in $\mathrm{IL}-1 \mathrm{Ra}^{-/-}$mice at 8 months post-BMT (14\%, 1 of 7 ), suggesting active $I L-1 R a^{-/-}$inflammation might be needed to cause femoral artery aneurysm.

Most aneurysm lesion sites in the mouse models are abdominal aorta, and those lesions are evoked by genetically defined approaches and/or by chemical means. The genetic approaches are spontaneous and engineered mutations. Mice that lack the gene for apolipoprotein $\mathrm{E}\left(\mathrm{Apo} \mathrm{E}^{-/-}\right)$develop atherosclerotic plaques in the aortic wall and showed subsequent fragmentation of the elastic lamellae in the media, and pseudomicroaneurysms. ${ }^{25}$ The $A p o E^{-1-}$ mouse provides an attractive model because the aneurysms are associated with atherosclerosis, but they have not yet been shown to produce progression aortic dilation. ${ }^{25}$ Recently, many studies have shown infusion of angiotensin $I I$ into $A p o E^{-1-}$ mice leads to the production of AAA. ${ }^{25-29}$ Delivery of angiotensin II leads to AAAs in the suprarenal region within the $\sim 21$ - to 28 -day infusion period. $25,27,29$ This study demonstrates that IL-1Ra deficiency in hematopoietic cells and T cells disrupts immune system homeostasis and may decrease tolerance for arterial wall components, resulting in the development of autoimmunity and inflammation. Consequently, inflammation induces spontaneous femoral artery aneurysms (but not AAA) in mice lacking interventions such as drugs or surgery. ${ }^{25-29}$ Thus, $I L-1 R a^{-1-}$ mice provide easy observations as they age and diminish the mortality that typically follows surgical procedures. Furthermore, none of the small animal aneurysm models reproduces the complex intraluminal thrombosis that is a prominent feature of most human aneurysms. ${ }^{26}$ Interestingly, our $I L-1 R a^{-1-}$ mice demonstrated several thrombosed large aneurysms of femoral and/or popliteal arteries at 10 months of age (24\%, 7 of 29). There is evidence to suggest that intraluminal thrombus affects aneurysm expansion in the human disease, ${ }^{30}$ possibly by acting as a reservoir of proteolytic activity or impeding the oxygenation of the aortic wall. ${ }^{31}$ Taken together, because $/ L-1 R a^{-/-}$mice contain the entire spectrum of lesions observed during inflammatory aneurysm, this mouse model likely will provide numerous opportu- 
nities to study the pathogenesis and therapy of inflammatory aneurysm.

\section{Acknowledgments}

We thank the members of the animal institute for assistance in animal care and Karen Williams for excellent editorial assistance.

\section{References}

1. Thompson RW: Basic science of abdominal aortic aneurysms: emerging therapeutic strategies for an unresolved clinical problem. Curr Opin Cardiol 1996, 11:504-518

2. Slifka MK, Whitton JL: Clinical implications of dysregulated cytokine production. J Mol Med 2000, 78:74-80

3. Benelli R, Lorusso G, Albini A, Noonan DM: Cytokines and chemokines as regulators of angiogenesis in health and disease. Curr Pharm Des 2006, 12:3101-3115

4. Moriwaki T, Takagi Y, Sadamasa N, Aoki T, Nozaki K, Hashimoto N: Impaired progression of cerebral aneurysms in interleukin-1 $\beta$-deficient mice. Stroke 2006, 37:900-905

5. Sadek M, Hynecek RL, Goldenberg S, Kent KC, Marin ML, Faries PL: Gene expression analysis of a porcine native abdominal aortic aneurysm model. Surgery 2008, 144:252-258

6. Dinarello CA: Interleukin-1, interleukin-1 receptors and interleukin-1 receptor antagonist. Int Rev Immunol 1998, 16:457-499

7. Dinarello CA: The role of the interleukin-1-receptor antagonist in blocking inflammation mediated by interleukin-1. N Engl J Med 2000, 343:732-734

8. Tait WF, Vohra RK, Carr HMH: True profunda femoris aneurysms: are they more dangerous than other atherosclerotic aneurysms of the femoro-popliteal segment? Ann Vasc Surg 1991, 5:92-95

9. Yamamoto N, Unno N, Mitsuoka H, Uchiyama T, Saito T, Kaneko H, Nakamura S: Clinical relationship between femoral artery aneurysms and arteriomegaly. Surg Today 2002, 32:970-973

10. Pappas G, Janes JM, Bernatz PE, Schirger A, Minn R: Femoral aneurysms. JAMA 1964, 190:489-93

11. Chan $\mathrm{O}$, Thomas $\mathrm{M}$ : The incidence of popliteal artery aneurysms in patients with arteryomegaly. Clin Radiol 1990, 41:185-9

12. Rigdon EE, Monajjem N: Aneurysms of the superficial femoral artery: a report of two cases and review of the literature. J Vasc Surg 1992, 16:790-3

13. Papadoulas S, Skroubis G, Marangos MN, Kakkos SK, Tsolakis JA: Ruptured aneurysms of superficial femoral artery. Eur J Vasc Endovasc Surg 2000, 19:430-2

14. Horai R, Asano M, Sudo K, Kanuka H, Suzuki M, Nishihara M, Takahashi $\mathrm{M}$, Iwakura $\mathrm{Y}$ : Production of mice deficient in genes for interleukin (IL)-1a, IL-1b, IL-1a/b, and IL-1 receptor antagonist shows that $\mathrm{IL}-1 \mathrm{~b}$ is crucial in turpentine-induced fever development and glucocorticoid secretion. J Exp Med 1998, 187:1463-1475
15. Isoda K, Shiigai M, Ishigami N, Matsuki T, Horai R, Nishikawa K, Kusuhara M, Nishida Y, Iwakura Y, Ohsuzu F: Deficiency of interleukin-1 receptor antagonist promotes neointimal formation after injury. Circulation 2003, 108:516-518

16. Isoda K, Sawada S, Ayaori M, Matsuki T, Horai R, Kagata Y, Miyazaki K, Kusuhara M, Okazaki M, Matsubara O, Iwakura Y, Ohsuzu F: Deficiency of interleukin-1 receptor antagonist deteriorates fatty liver and cholesterol metabolism in hypercholesterolemic mice. J Bio Chem 2005, 280:7002-7009

17. Hedrick CC, Castellani LW, Warden CH, Puppione DL, Lusis AJ: Influence of mouse apolipoprotein A-II on plasma lipoproteins in transgenic mice. J Biol Chem 1993, 20676-20682

18. Isoda K, Matsuki T, Kondo H, Iwakura Y, Onsuzu F: Deficiency of interleukin-1 receptor antagonist induces aortic valve disease in BALB/c mice. Arterioscler Thromb Vasc Biol 2010, 30:708-715

19. Libby $P$ : Inflammation in atherosclerosis. Nature $2002,420: 868-874$

20. Junqueira LC, Cossermelli W, Brentani R: Differential staining of collagens type I, II and III by Sirius Red and polarization microscopy. Arch Histol Jpn 1978, 41:267-274

21. Satta J, Haukipuro K, Kairaluoma MI, Juvonen T: Aminoterminal propeptide of type III procollagen in the follow-up of patients with abdominal aortic aneurysms. J Vasc Surg 1997, 25:909-915

22. Thompson RW, Parks WC: Role of matrix metalloproteinases in abdominal aortic aneurysms. Ann N Y Acad Sci 1996, 800:157-174

23. Muzio M, Stockwell BR, Stennicke HR, Salvesen GS, Dixit VM: An induced proximity model for caspase-8 activation. J Biol Chem 1998, 273:2926-30

24. Tedgui A, Mallat Z: Anti-inflammatory mechanisms in the vascular wall. Circ Res 2001, 88:877-887

25. Choke E, Cockerill GW, Dawson J, Howe F, Wilson WR, Loftus IM Thompson MM: Vascular endothelial growth factor enhances angiotensin II-induced aneurysm formation in apolipoprotein E-deficient mice. J Vasc Surg 2010, 52:159-166

26. Carrell TW, Smith A, Burnand KG: Experimental techniques and models in the study of the development and treatment of abdominal aortic aneurysm. Br J Surg 1999, 86:305-12

27. Jones A, Deb R, Torsney E, Howe F, Dunkley M, Gnaneswaran Y, Gaze D, Nasr H, Loftus IM, Thompson MM, Cockerill GW: Rosiglitazone reduces the development and rupture of experimental aortic aneurysms. Circulation 2009, 23;119:3125-32

28. Gopal K, Kumar K, Nandini R, Jahan P, Kumar MJ: High fat diet containing cholesterol induce aortic aneurysm through recruitment and proliferation of circulating agranulocytes in apoE knock out mice model. J Thromb Thrombolysis 2010, 30:154-63

29. Daugherty A, Cassis LA: Mouse models of abdominal aortic aneurysms. Arterioscler Thromb Vasc Biol 2004, 24:429-434

30. Wolf YG, Thomas WS, Brennan FJ, Goff WG, Sise MJ, Bernstein EF: Computed tomography scanning findings associated with rapid expansion of abdominal aortic aneurysms. J Vasc Surg 1994, 20: 529-38

31. Vorp DA, Federspiel WJ, Webster MW: Does laminated intraluminal thrombus within abdominal aortic aneurysm cause anoxia of the aortic wall? J Vasc Surg 1996, 23:540-541 